\title{
The Hamilton-Jacobi treatment for non-abelian Chern-Simons system
}

\author{
November 2, 2018
}

\author{
S. I. Muslih \\ Department of Physics, Al-Azhar University, Gaza, Palestine
}

\begin{abstract}
The non-abelian Chern-Simons field interacting with $N$ component complex field is treated as a constrained system using the HamiltonJacobi approach. The reduced phase space Hamiltonian density is obtained with out introducing Lagrange multipliers and with out any additional gauge fixing condition. The quantization of this system is also discussed.
\end{abstract}




\section{Introduction}

In an earlier work [1], it was shown that the canonical method [Q], [3] leads to obtain the equations of motion and the correct reduced phase space Hamiltonian for an abelian Chern-Simons field system interacting with scalar field without using any gauge fixing conditions.

In the present paper we summarize our results for a similar demonstration for the non-abelian Chern-Simons term. In fact, this system has been discussed by several authors [4, 5, 6] and it was shown in reference [6], that the gauge conditions consistent with equations of motion can be chosen. We show in this work that using the Hamiton-Jacobi method [2, 阴, the reduced phase space Hamiltonian density is obtained without using any gauge fixing conditions. Besides, we discuss the quantization of this system using the Hamilton-Jacobi method.

\section{The Hamilton-Jacobi method of constrained systems}

In this section we will briefly review the Hamilton-Jacobi method [Q, [3] for studying the constrained systems.

This formulation leads us to obtain the set of Hamilton-Jacobi partial differential equations [HJPDE] as follows:

$$
\begin{aligned}
& H_{\alpha}^{\prime}\left(t_{\beta}, q_{a}, \frac{\partial S}{\partial q_{a}}, \frac{\partial S}{\partial t_{\alpha}}\right)=0, \\
& \alpha, \beta=0, n-r+1, \ldots, n, \\
& a=1, \ldots, n-r,
\end{aligned}
$$

where

$$
H_{\alpha}^{\prime}=H_{\alpha}\left(t_{\beta}, q_{a}, p_{a}\right)+p_{\alpha},
$$

and the Hamiltonian $H_{0}$ is defined as

$$
\begin{aligned}
& H_{0}=p_{a} w_{a}+\left.p_{\mu} \dot{q}\right|_{p_{\nu}=-H_{\nu}} \\
& -L\left(t, q_{i}, \dot{q_{\nu}}, \dot{q_{a}}=w_{a}\right), \\
& \quad \mu, \nu=n-r+1, \ldots, n .
\end{aligned}
$$


The equations of motion are obtained as total differential equations in many variables as follows [3]:

$$
\begin{aligned}
& d q_{a}=\frac{\partial H_{\alpha}^{\prime}}{\partial p_{a}} d t_{\alpha}, \quad d p_{a}=-\frac{\partial H_{\alpha}^{\prime}}{\partial q_{a}} d t_{\alpha} \\
& d p_{\beta}=-\frac{\partial H_{\alpha}^{\prime}}{\partial t_{\beta}} d t_{\alpha} \\
& d z=\left(-H_{\alpha}+p_{a} \frac{\partial H_{\alpha}^{\prime}}{\partial p_{a}}\right) d t_{\alpha}
\end{aligned}
$$

where $z=S\left(t_{\alpha} ; q_{a}\right)$. The set of equations (4-6) is integrable if [2]

$$
d H_{0}^{\prime}=0, \quad d H_{\mu}^{\prime}=0 .
$$

If condition (7) are not satisfied identically, one considers them as new constraints and again testes the consistency conditions. Hence, the canonical formulation leads to obtain the set of canonical phase space coordinates $q_{a}$ and $p_{a}$ as functions of $t_{\alpha}$, besides the canonical action integral is obtained in terms of the canonical coordinates. The Hamiltonians $H_{\alpha}^{\prime}$ are considered as the infinitesimal generators of canonical transformations given by parameters $t_{\alpha}$ respectively.

\section{Non-abelian Chern-Simons as a constrained system}

The Lagrangian density for Chern-Simons field interacting a scalar field in $2+1$ dimensions is given by

$$
\begin{aligned}
& \mathcal{L}=\left[D_{\mu} \Phi_{\alpha}\right]^{\dagger}\left[D^{\mu} \Phi_{\alpha}\right] \\
& +\frac{\kappa}{4 \pi} \epsilon^{\mu \nu \lambda} \operatorname{Tr}\left[A_{\mu} \partial_{\nu} A_{\lambda}-\frac{2}{3} A_{\mu} A_{\nu} A_{\lambda}\right],
\end{aligned}
$$

where $\Phi$ is $N$ - component scalar field which transforms according to funda-

mental relation of Lie group $S U(N)$ and the parameter $\kappa$ plays the role of a coupling constant. Here

$$
\begin{aligned}
& D_{\mu}=\partial_{\mu}-i e A_{\mu}, \\
& A_{\mu}=i g T_{a} A_{\mu}^{a},
\end{aligned}
$$


and $T^{a}$ are the generators of the compact Lie group $S U(N)$

$$
\begin{gathered}
{\left[T^{a}, T^{b}\right]_{-}=i f^{a b c} T^{c},} \\
{\left[T^{a}, T^{b}\right]_{+}=d^{a b c} T^{c}+\frac{1}{N} \delta^{a b},} \\
\operatorname{Tr}\left(T^{a} T^{b}\right)=\frac{1}{2} \delta^{a b},
\end{gathered}
$$

where $d^{a b c}$ is totally symmetric tensor in the indices $a, b, c$ while $f^{a b c}$ are the usual structure constants of $S U(N)$.

Canonically conjugated momenta for $\Phi$ and $\Phi^{\dagger}$ are called $P$ and $P^{\dagger}$, where

$$
P_{\alpha}=\dot{\Phi}_{\alpha}^{\dagger}+i g \Phi_{\beta}^{\dagger} A_{0}^{a} T_{\beta \alpha}^{a}
$$

The canonical momenta $\Pi_{\rho}^{a}$ conjugated to $A_{\rho}^{a}$ are defined as

$$
\Pi_{\rho}^{a}=-\frac{\kappa}{8 \pi} g^{2} \epsilon^{\mu 0 \rho} A_{\mu}^{a}
$$

The primary constraints are obtained as follows:

$$
\begin{aligned}
\mathcal{H}_{0}^{\prime a} & =\Pi_{0}^{a}=0 \\
\mathcal{H}_{1}^{\prime a} & =\Pi_{1}^{a}+\frac{\kappa}{8 \pi} g^{2} A_{2}^{a}=0, \\
\mathcal{H}^{\prime a} & =\Pi_{2}^{a}-\frac{\kappa}{8 \pi} g^{2} A_{1}^{a}=0 .
\end{aligned}
$$

The canonical Hamiltonian density can be written as

$$
\begin{aligned}
& \mathcal{H}_{0}^{c}=P_{\alpha}^{\dagger} P_{\alpha}+\left(\partial_{i} \Phi_{\alpha}^{\dagger}\right)\left(\partial^{i} \Phi_{\alpha}\right) \\
& +A_{0}^{a} j_{0}^{a}+A_{i}^{a} j_{i}^{a}+g^{2} \Phi_{\beta}^{\dagger} A_{i}^{a} T_{\beta \alpha}^{a} T_{\alpha \gamma}^{b} A_{i}^{b} \Phi_{\gamma} \\
& +\frac{\kappa}{8 \pi} g^{2} \epsilon^{i j}\left[A_{0}^{a} \partial_{i} A_{j}^{a}+A_{i}^{a} \partial_{j} A_{0}^{a}\right] \\
& -\frac{\kappa}{8 \pi} g^{3} \epsilon^{i j} A_{0}^{a} A_{i}^{c} A_{j}^{b} f^{a b c}
\end{aligned}
$$

where

$$
\begin{aligned}
& j_{0}^{a}=i g\left[P_{\alpha} \Phi_{\beta}-P_{\alpha}^{\dagger} \Phi_{\beta}^{\dagger}\right] T_{\beta \alpha}^{a} \\
& j_{i}^{a}=-i g\left[\left(\partial_{i} \Phi_{\alpha}^{\dagger}\right) \Phi_{\beta}-\Phi_{\beta}^{\dagger}\left(\partial_{i} \Phi_{\alpha}\right)\right] T_{\beta \alpha}^{a}
\end{aligned}
$$


Now, the canonical method leads us to obtain the set of Hamilton-Jacobi partial differential equations as follows:

$$
\begin{aligned}
& {H^{\prime c}}_{0}=\int d^{2} x\left(p_{0}+P_{\alpha}^{\dagger} P_{\alpha}+\left(\partial_{i} \Phi_{\alpha}^{\dagger}\right)\left(\partial^{i} \Phi_{\alpha}\right)\right. \\
& +A_{0}^{a} j_{0}^{a}+A_{i}^{a} j_{i}^{a}+g^{2} \Phi_{\beta}^{\dagger} A_{i}^{a} T_{\beta \alpha}^{a} T_{\alpha \gamma}^{b} A_{i}^{b} \Phi_{\gamma} \\
& +\frac{\kappa}{8 \pi} g^{2} \epsilon^{i j}\left[A_{0}^{a} \partial_{i} A_{j}^{a}+A_{i}^{a} \partial_{j} A_{0}^{a}\right] \\
& -\frac{\kappa}{8 \pi} g^{3} \epsilon^{i j} A_{0}^{a} A_{i}^{c} A_{j}^{b} f^{a b c}=0 \\
& {H^{\prime a}}^{a}=\int d^{2} x\left(\Pi_{0}^{a}\right)=0 \\
& H_{1}^{\prime a}=\int d^{2} x\left(\Pi_{1}^{a}+\frac{\kappa}{8 \pi} g^{2} A_{2}^{a}\right)=0 \\
& H_{2}^{\prime a}=\int d^{2} x\left(\Pi_{2}^{a}-\frac{\kappa}{8 \pi} g^{2} A_{1}^{a}\right)=0 .
\end{aligned}
$$

To check whether this model is integrable or not we have to consider the integrabilty conditions (7). In fact, the total variations of the constraints (22-25) lead to obtain the total variations of the fields $A_{1}^{a}$ and $A_{2}^{a}$ in terms of $d t$. Besides, the total variations of ${H^{\prime}}_{0}^{a}$ yield

$$
d H_{0}^{\prime a}=H_{3}^{\prime a} d t
$$

where ${H^{\prime}}_{3}$ are given by

$$
H_{3}^{\prime a}=j_{0}^{a}+\frac{\kappa}{4 \pi} g^{2} \epsilon^{i j}\left[\partial_{i} A_{j}^{b}-\frac{g}{2} A_{i}^{c} A_{j}^{e} f^{a b c}\right] .
$$

The total variations of ${H^{\prime}}_{3}^{a}$ vanish and no further constraints arise.

Now the reduced phase space Hamiltonian density is calculated as

$$
\begin{gathered}
\mathcal{H}_{0}^{c_{r}}=\left.\mathcal{H}_{0}^{c}\right|_{H_{3}^{\prime a}}=P_{\alpha}^{\dagger} P_{\alpha}+\left(\partial_{i} \Phi_{\alpha}^{\dagger}\right)\left(\partial^{i} \Phi_{\alpha}\right) \\
+A_{i}^{a} j_{i}^{a}+g^{2} \Phi_{\beta}^{\dagger} A_{i}^{a} T_{\beta \alpha}^{a} T_{\alpha \gamma}^{b} A_{i}^{b} \Phi_{\gamma} .
\end{gathered}
$$

Finally, to obtain the path integral quantization [7] of this model, we have to discuss the integrability conditions in terms of the action. In fact, Eq. (6) yields

$$
d S=\int d^{2} x\left(-\mathcal{H}_{\alpha}+p_{a} \frac{\delta \mathcal{H}_{\alpha}^{\prime}}{\delta p_{a}}\right) d t_{\alpha}
$$


Making use of (29) and (22-25), we obtain the action of the form

$$
\begin{aligned}
S=\int & d^{2} x\left(-\mathcal{H}_{0}^{c} d t+\Pi_{0}^{a} d A_{0}^{a}+\Pi_{1}^{a} d A_{1}^{a}\right. \\
& \left.+\Pi_{2}^{a} d A_{2}^{a}+P_{\alpha} d \Phi_{\alpha}+P_{\alpha}^{\dagger} d \Phi_{\alpha}^{\dagger}\right) .
\end{aligned}
$$

Using the definition of the canonical momenta, one can recover the original action

$$
S=\int d^{3} x \mathcal{L}
$$

where $\mathcal{L}$ is exactly the same as the first-order Lagrangian (1) in the symplectic formalism.

\section{Conclusion}

The basic idea of Dirac's method [8] to investigate the non-abelian ChernSimons system is to consider the total Hamiltonian composed by adding the constraints multiplied by Lagrange multipliers to the canonical Hamiltonian. In order to derive the equations of motion, one needs to redefine these unknown multipliers in an arbitrary way [ [ . 5, 6]. However, in the Hamilton-Jacobi method [2, 3], there is no need to introduce Lagrange multipliers to the canonical Hamiltoian as well as no need to use any gauge fixing conditions.

Unlike conventional methods one can perform the path integral quantization of this system using the canonical path integral method [7] to obtain the action directly without considering any Lagrange multipliers and without any gauge fixing conditions.

\section{References}

[1] S. I. Muslih, math-ph/0101021.

[2] S. I. Muslih and Y. Guler, Nuovo Cimento B, 110 (1995) 307; 113 (1998) 277.

[3] Y. Guler, Nuovo Cimento B, 107(1992) 1389; 107 (1992) 1143.

[4] F. Ferrari, Phys. Rev. D. 50, (1994) 7578. 
[5] S. Deser, R. Jackiw and S. Templeton, Ann. Phys. (N. Y.) 140 (1984) 372.

[6] K. Dasgupta, M. Sami, P. Sharan and A. Mehra and, Nuvo Cimento B, 114 (1999) 1219.

[7] S. I. Muslih, Nuovo Cimento B, 115 (2000) 7; 115 (2000) 1; S. I. Muslih, Hadronic Journal, 23 (2000) 203; Hadronic J. Supp., 15 (2000) 461; S. I. Muslih, H. El-Zalaan and F. El-Sabaa, Inter. J. of Theor. Phys., 39 (2000) 2495; S. I. Muslih, math-ph/0009015.

[8] P. A. M. Dirac, Lectures on Quantum Mechanics, Belfer Graduate School of Science, Yehiva University (A cademic Press, New York) 1964. 